\title{
Face transplantation for the blind: more than being blind in a sighted world
}

\author{
Joseph Lee
}

\section{Correspondence to \\ Dr Joseph Lee, College of Humanities, Arts and Social Sciences, Flinders University, Adelaide, SA 5001, Australia joseph.lee@flinders.edu.au}

Received 4 September 2017 Revised 8 January 2018 Accepted 6 February 2018 Published Online First 24 February 2018
Check for updates

To cite: Lee J. J Med Ethics 2018:44:361-365.
ABSTRACT

Face transplantation (FT) is a landmark in reconstructive surgery involving vascularised composite allotransplantation. A recent issue of $\mathrm{FT}$ for patients who are blind has arisen. Some bioethicists recommend not excluding a patient who is blind, as this may amount to discrimination. From an ethical standpoint, FT for those with blindness is appropriate in selected candidates. This article seeks to add to the clinical evidence supporting FT for those with blindness by detailing a complementary psychosocial perspective. Currently, there is little relevant research about the subjectivity of the blind. This is critical since the arguments against FT for the blind refer to their inability to see their face and to view the reaction of others to their disfigured faces. We begin with a brief look at examples of FT involving blindness and associated arguments. The next part is a multidisciplinary investigation of the experiences of the blind. These are gleaned from a close reading of the literature and drawing inferences, as direct studies are rare. The discussion analyses identity themes of the blind in relation to their faces: as they experience it; the face they wish to show to the world; and how others perceive and react to their face in a saturated environment of imagery and visual communication. Disability and the blind person's experience of faces are well-founded considerations for medical practitioners and ethics boards in the process of FT decision-making.

\section{INTRODUCTION}

Face transplantation (FT) is a promising advance in regenerative and restorative surgery, involving vascularised composite allotransplantation which transfers living tissue from a donor to a recipient including skin, bone, muscle, tendon and nerves. A recent issue of FT for patients who are blind has emerged. Total or complete bilateral blindness is an absolute contraindication in one programme. ${ }^{2}$ Yet elsewhere at least three face transplants performed have involved patients with total bilateral blindness (TBB). ${ }^{3}$

Some bioethicists advise not to exclude such patients who are blind, as this may amount to discrimination based on disability. ${ }^{4}$ With the success of TBB FT, one thought is that it is unethical to use $\mathrm{TBB}$ as an automatic exclusion criterion for FT. It is argued that a patient with TBB who has the capacity to benefit from FT ought not to be denied what the transplant potentially offers, for example, the ability to eat and drink by mouth. ${ }^{3}$ Nonetheless, concerns about including a patient who is completely blind in a face transplant protocol are also linked with rehabilitation and post-transplant care.
This article aims to add to the clinical evidence supporting FT for those with $\mathrm{TBB}^{3}$ by detailing a psychosocial perspective which also favours FT for patients who are completely blind. From an ethical standpoint, FT for those with TBB is appropriate in selected candidates. ${ }^{35}$ Scholars usually apply the normative principles of justice and non-discrimination to the delivery of medical care. However, there is very little research about the subjectivity of the blind. This is critical since the arguments against FT for patients who are blind with TBB, as we shall consider shortly, refer to their inability both to see their face and to view the reaction of others to their disfigured faces. By examining their situation, we can derive patient-centred themes of blind face identity which enhance the arguments in favour of TBB FT and answer the objections.

We begin with a brief look at examples of FT involving TBB and associated arguments. The next part is a multidisciplinary investigation of the experiences of the blind, concentrating on the patient as subject. These are gleaned from a close reading of the literature and drawing inferences, as direct studies are rare. The discussion analyses complementary themes for the blind in relation to their faces: as they experience it; the face they wish to show to the world; and how others perceive and react to their face in a saturated environment of imagery, visual communication and face recognition.

\section{CASES AND POSITION}

There are three cases of FT recipients with TBB. One patient was a 24-year-old man who suffered accidental high-voltage electrical burn injuries to the entire face and the left hip, with facial defect of whole face and extended into the scalp, causing blindness. ${ }^{6}$ Another patient was a 57 -year-old woman who was savagely mauled by a large animal, resulting in devastating multiple composite tissue losses including most of the central face and both hands, and complicated by blindness. A separate study investigated the psychological, marital and family functioning of a blind 54-year-old patient and his partner after FT, and found positive psychosocial outcomes. ${ }^{7}$

Overall there were expected poor clinical outcomes with a conventional reconstructive approach. ${ }^{6}$ Instead, FT offered genuine functional improvements to the patients' conditions such as no nose, lips, teeth, oral aperture of $2 \mathrm{~cm}$ and inability to breathe through the nose. Yet, the functional and the aesthetic linked. Allotransplantation of the face was considered an option to 
re-establish vital functions and restore aesthetics in a one-stage procedure. $^{7}$

Moreover, these patients have adventitious blindness and, in contrast to those with lifelong congenital blindness, have to contend with other psychosocial issues concerning the loss of vision, for example, threats to self-esteem, possibly mourning, depression and coping. There is also the social stigma of blindness and responses, for example, a veteran who had loss of vision was more traumatised by the negative reactions of family members than by his loss of sight. ${ }^{8}$

Carty et al published a position paper supporting FT in the blind. ${ }^{5}$ It uncovered several inferred reservations associated with the view that complete bilateral blindness ought to be reckoned an absolute contraindication to facial allotransplantation. The identity objections can be synthesised into four: that benefits are mostly cosmetic; inability to see the disfigured face; inability to appreciate visual aesthetics; and inability to appreciate interpersonal responses. Most focus on the individual's disability. These points will be re-evaluated in view of the lesser known psychosocial factors.

\section{FACES AND THE BLIND}

The thinking needs to widen beyond clinical studies to learn from the blind as a specific patient group who are not excluded from the benefits of FT. There are six noteworthy and transplant-relevant considerations to be discovered by inquiring into cognition, self-expression, non-verbal communication, body image and appearance, neuroscience and assistive technology. These unique experiences of faces in the blind are not normally pondered in discussion among the sighted, but they are revealing and can bring a welcome patient-impact discourse to inform ethical analysis.

\section{Blind sensing and perceiving the face}

Scholars have found that people who have been totally blind from birth have the same strong visual understanding of race as sighted people.' Blind people use their hearing sense, for example, 'When it is noisy, I do not see you speaking to me.'10 Although sighted people perceive nuances of mood primarily by changes in facial expressions of the speaker, instead for the blind it is by mutations of tone exclusively.

For many years, one blind man knew the time by taking a large watch, without its glass cover, and rapidly touching its hands. ${ }^{11}$ As he 'read' the clock face by touch, so too many blind people seek to interpret human faces proprioceptively.

In contrast, some note how there are photographs of Helen Keller, a remarkable deaf-blind person, touching the faces of celebrities, for example, Eleanor Roosevelt and Charlie Chaplin. ${ }^{12}$ Another view is that exploring a stranger's face with the hands breeches social norms in ways that reinforce the blind people's outsider status. Such touching would not be able to read the expression because touching would modify the face, inhibit or erode the expression. ${ }^{4}$

Generally, those without sight are not 'blind' to faces but have alternative insights: conceptually, tactilely and mentally. Even if the blind choose not to feel people's faces, it manifests respect for others and etiquette. Nevertheless, this desire to capture information expressed on faces indicates that the blind recognise the importance of the face and its power to express identity. If the idiom 'a picture is worth a thousand words' holds true, then seeing a face must be enlightening. But the blind apprehend facial connotations in a more specialised, time-intensive way requiring different cognitive routes compared with the sighted. For both the face has meaning.

\section{Expressing the blind face}

Blind people can also represent faces. Drawing and the visual arts involve cognitive and motor skills. The experimental evidence supports the hypothesis that mental representations of objects can be produced by haptic experiences which are transformed into representations that can be understood by sight. One totally blind early-teenage girl could draw human figures similar to drawings by sighted $10-12$ year-olds. ${ }^{13}$ Likewise, adolescents blind from birth can develop concepts of faces as expressed through clay modelling. ${ }^{14}$

For the sighted, to make a drawing of an object means converting the information received by eyes into a new form that will control the muscles of the hands. ${ }^{15}$ Neuroimaging experiments suggest that choosing a figure activates regions of the temporal lobe linked with what an object is, while choosing locations activates parietal regions linked with where an object is. Thus, 'drawing' an object activates regions that are similar to those concerned with seeing an object.

Summing up, in drawing faces the research highlights that the blind, from childhood onwards, can recognise the concept of faces, acquired through other channels; and expressed manually. All this demonstrates that the blind possess a working facial knowledge; they recognise the phenomenological importance of the face for human identity.

\section{Communicating through the blind face}

Face-to-face communication involves aspects of the face. Blind persons turn their face or an ear towards sounds, presuming the blind have learnt this through social means. ${ }^{16}$ For some blind people, the smile is physical in that it generates a sensation inside the self, and in the throat, a bubbly feeling...You can feel your face twist and certain muscles relax so you know intellectually that his changes the shape of your face,' whereas, 'You would have to ask someone how much my face reveals when I am sad because I am not necessarily so aware of it as in smiling. ${ }^{17}$ This blind person noted how some of the blind cannot see and appreciate the importance of the face and manner and body language. Blind children smile when happy but do not make a social smile naturally when it is expected.

In a seeing world, facial characteristics, especially the eyes, are essential, as they facilitate person-to-person conversations. The blind experience a 'facial deficit' so to speak in their ability to communicate affective dimensions non-verbally. They depend on alternative means such as face muscle movements, mouth, oral language, sounds, head positioning and gestures. All this manifests the significance of the face for blind people, and their awareness of the face's contribution to interpersonal communication.

\section{Blind social self-perception: appearance and public opinions about the face}

Exploring body image and the blind provides insights for identity. Interviews with blind people revealed that they are concerned about their physical appearance. It is because of a customary knowledge among the blind that although they cannot see themselves, other can and do see them. ${ }^{18}$

Yet, the inability of the blind to see images of thin attractive women means that blind women may accord less significance to appearance and may be less likely to internalise the thin ideal as their personal goal. ${ }^{18}$ Some research found that sighted women felt significantly more dissatisfied with their body and restrained 
their eating significantly more than blind women, and that sighted women were significantly more aware of the thin ideal and endorsed that as a personal goal significantly more than congenitally blind women. ${ }^{19}$

These feminine images, which are manifested visually in magazines, television and advertising, are not available to blind women. Nonetheless, some blind women use appearance as a powerful tool to defy the cultural labels of 'blind' and 'disabled'. One study quoted from Ayelet, a 33-year-old congenitally blind woman, who practised a highly groomed femininity and wears make-up and earrings at work, 'I want people to look at me and say 'Walla,' she's ok. Her being blind doesn't make her ugly and it's not like she doesn't groom herself... What matters is that they look at me and don't see a damaged and ugly person. ${ }^{20}$ Roni, a 27-year-old congenitally blind concurs: '...no, I don't see myself in the mirror but it's important for me to look good. So, you know, going to the esthetician, plucking my eyebrows, even going to the hairdresser, to highlight my hair. ${ }^{20}$

It is also observed how actors, models and many adolescents spend time in front of mirrors practising facial expressions. In ordinary life, the blind may feel compelled to put on a face, for example, in anticipating being told a secret one blind person thinks about her face. 'I was suddenly very conscious of my facial expression, anxious that my face should not look shocked or judgmental. I tried to relax all the muscles in my face, except those holding up my eyelids-lowered lids denote indifference. ${ }^{12}$

For some blind people, daily grooming and a sense of beauty are empowering. It entails accepting the influential visual concepts of appearance and attractiveness. The blind who intentionally engage in face enhancement behave as if they were sighted. Therefore, they identify with the ruling appearance culture in non-visual ways. The natural alternative to make-up for faces is the faces the blind make to others: the kinaesthetic face presentations, which have inherent competence to communicate interior states of mind (see the Communicating through the blind face section).

\section{Assistance from nature: faces, blindness and the brain}

The experiences of the blind and their faces are supported by neuroscience. Nearly one-quarter of the brain is normally devoted to processing visual information, for example, reading text. ${ }^{21}$ Yet in congenitally blind people, most of the 'visual' cortex responds strongly to tactile and auditory input instead of visual stimuli, a phenomenon called cross-modal plasticity. Some early 20th-century Irish and British literatures portray blindness as conducive to intimacy, as the absence of vision partly directs more attention to touch and hearing, two senses understood to cultivate human attachment. ${ }^{22}$

The research data confirm compensation for the blind. Scientists are interested in whether the ability to facially communicate physical and psychological distress, for example, pain, represents innate and biologically prepared programmes or whether it requires visual learning. A study of sighted and congenitally blind individuals found that the range of evoked facial expressions was comparable in both groups. ${ }^{23}$ However, blind individuals were less able to facially encode different intensities of experimental pain. Furthermore, blind individuals were less able to voluntarily modulate their pain expression. The study concluded that the repertoire of facial muscles activated during pain is biologically prepared. However, these biologically prepared algorithms need to be customised and this appears to require visual learning in the early childhood to generate different facial expressions attuned to the situational requirements.
Blind faces are active. A study compared the expressions of congenitally and non-congenitally blind athletes in the 2004 Paralympic Games with each other and with those produced by sighted athletes in the 2004 Olympic Games. ${ }^{24}$ It found no differences between congenitally blind, non-congenitally blind and sighted athletes on the level of individual facial actions and also in facial emotion configurations. While blind athletes did produce more overall facial activity, these differences were isolated to eye and head movements, and not the facial musculature. The blind athletes' expressions did differ where they had won or lost a medal match at three different points in time, with no cultural differences in expression.

These findings offer evidence that the production of spontaneous facial expressions of emotion is not reliant on observational learning, yet demonstrates a learnt component in the social management of expressions, even among blind individuals. ${ }^{24}$ The blind athletes, particularly those born blind, could not possibly have learnt to produce those precise facial configurations from modelling the faces of others in culturally stable settings. The study suggests there may be ways of understanding the potential mechanisms where individuals learn to regulate their emotional displays, for example, general reinforcement. These do not involve the sense of sight, implying that visual observation may not be necessary for such learning.

Summing up, nature bestows assistance to the blind and their faces through other sensory modalities and neuroplasticity. Where the blind are at risk of being marginalised a sighted world, human ingenuity may help.

\section{Assistive technology for blindness}

The social-cultural limitations of loss of sight can be aided by assistive technology designed to facilitate non-verbal communication. For instance, EmoAssist has particular functionalities used to predict behavioural expressions in two-way conversations, for example, a closed lip smile, an open lip smile, yawn, looking away, and so on, and three-dimensional affective dimensions, for example, valence, from facial features and head pose so as to provide the correct auditory feedback. ${ }^{25}$

Assistive technologies show that in concept designs and realworld applications, recognising faces is critical to human society and can help in forming the self-concept of faces for the blind. It also fosters their agency in society.

\section{ANSWERING OBJECTIONS}

It is timely to re-examine the four identity objections to FT in the blind. ${ }^{5}$ First, that benefits are mostly cosmetic. This is significant in contemporary healthcare characterised by evidence and cost-benefit and cost-effectiveness evaluations. Now, there are few precise references to cosmetic surgery in a blind person except for one augmentation mammaplasty and abdominal lipectomy. ${ }^{26}$ For surgeons, the beginning of intervention will be an objective abnormality with a major component of functional loss due to congenital absence, trauma or tumour extirpation. Plastic surgery will have an important component of reconstruction. ${ }^{27}$ Nevertheless, FT is reconstructive surgery with functional outcomes.

We think of how medical practitioners treat patients who are blind with melanoma on the face, or fractured jaw, or facial burn injury. The face is objective, for example, the site of trauma. Blindness is not a contraindication to treatment. Why is there hesitation for FT? Probably because it involves the face and not a condition or vital organ; it affects the whole person too for a lengthy period of time or a lifetime, for example, 
immunosuppression. There is also the pressing concern about full participation in postoperative care when assessing patients with TBB: absence of visual input to facilitate rehabilitation of the transplanted face, incapacity to visually self-monitor for signs of rejection and the need for assistance with daily medication compliance. ${ }^{6}$

Yet a damaged face is still a face. Disfigurement can provoke reactions for the sighted and the blind. It becomes part of blind people's identities and their subjective thinking about who they are. ${ }^{26}$ Severe facial disfigurement can contribute to social death, that is, social isolation and ostracism; a facial transplant can assist re-entry into a community, enhance one's social capacity and motivation to seek medical treatment to alter their situation. ${ }^{28}$

Second, the inability to see the disfigured face. The blind cannot see their own face. This is true. However, as explained above, blind individuals can feel their own face, and others see their faces. The blind have indirect non-visual perceptions of disfigurement: they experience it in the first person and interpersonally. The blind are conscious of what a face is: subjectively, kinaesthetically, tactilely, conceptually, socially...except visually.

The blind experiences of faces are authentically human even if the face is not seen. To set blindness as a contraindication for FT is to restrict FT to vision alone; consequently, it becomes an exclusively sight-specific medical advancement. This somewhat confines the nature and purpose of FT, thereby ignoring or discounting the sensing, perceiving and phenomenological lives of those without sight.

The third objection to FT for the blind is the inability to appreciate visual aesthetics. FT is intended for the sighted world and obviously there are questions raised about eligibility criteria for FT pertaining to those lacking sight. Yet the blind can smell, taste, hear sounds and feel surfaces: the appreciation of the world is still multisensory. The olfactory, gustatory, aural and tactile sensation conveys meaning through processing and integrating sensory information from multiple sensory modalities. The blind appreciate visual aesthetics through drawing faces, as well as in the imagination and creativity, for example, audio books can paint a word picture. The blind person still lives sensorially, perceptively and meaningfully and is a competent ethical agent.

Fourth, there is the objection of an inability to appreciate interpersonal responses. This is an understandable point. But as noted earlier, the blind have a firm grasp of race. ${ }^{9}$ They are situated in human societies which are often accommodating of visually impaired citizens. Blind people use corresponding non-verbal cues, for example, hearing tonal variation instead of facial expressions. The blind can use the muscular system to make faces and some highly value face make-up to be better involved with the visual world. With assistance from technology, the blind can better grasp facial responses in a slower but equivalent manner to the sighted.

\section{CONCLUSIONS}

The majority of humanity has five working senses; yet we cannot deny the experiences of the blind with respect to faces. They sense and perceive their faces, express their face and communicate through that face. The blind are conscious of public perceptions of the facial appearance. They are helped by nature and assistive technology to contribute to society.

Most objections to FT focus on the blind person's disability in a seeing world. ${ }^{5}$ Yet they participate individually, socially, culturally and ethically, like other human beings. Moreover, FT actually treats trauma or face disfigurement, not disability (blindness). Then ethically how is disability a contraindication?
Because the disability has potential to impede the rehabilitation processes. ${ }^{3}$ The implied assumption behind the objections to FT for the blind is that the sighted recipients have greater capacity to benefit, because they enjoy visual sensation and perception.

Otherwise it would be tantamount to denying a knee replacement to paraplegics with severe knee arthrosis because they are not able-bodied. Or refusing a hair transplant to a blind person with early-onset baldness; or even denying a wig or hat to blind chemotherapy patients losing their hair-all because the blind cannot see the effects of hair loss.

There is also the factor of resource allocation. Given that FT is complex and expensive surgery, it can be argued that those who benefit the most ought to be afforded access. Patients who can see the transplanted face would likely benefit more, for example, increase in self-esteem, compared with patients who are blind who cannot see the outcomes of surgery. However, this is countered by the psychosocial perspectives discussed, and other factors such as equity which involves fairness in the distribution of health across individuals. ${ }^{29}$ It has been demonstrated that people appreciate alternate methods of allocating health gains by fairness or equity, for example, to groups with disabilities.

With deeper investigation, the blind person's capacity to benefit needs thoughtful deliberation and reappraisal. Not simply from a non-discriminatory ethical norm but from a socially-immersed human identity perspective. Although visual perception is so prevailing, seeing is not the only sense. This counteracts the sighted argument against FT for the blind and philosophically questions the cultural reign of sight. Art therapists comment how a seeing person's notion of blindness is often dominated by the concept of the absence of seeing. ${ }^{14}$

Such a notion of blindness as 'not seeing' influences how blind people are trained in an institution and are its goals. Most staff are seeing people. The vital words are 'mobility' and 'practical life skills' which indicate the aims of this education: 'to orient toward and manage in a visual world where rules are set by visual people. A student's day is tightly planned and crammed with extra training to achieve these goals of independence and thus ultimately defeat, perhaps even deny, blindness.' 14

Interestingly, this situation is somewhat reminiscent to the Deaf Pride movement which refuses to contemplate paediatric cochlear implantation. ${ }^{30}$ There was a conviction on the part of deaf people that their lives and deafness had meaning for them. In the same way, to be independent of the media-driven visual culture, a blind person may purposely decide not to participate in the face beautification practices, or forego FT unless for absolute medical reasons. However, that comparison may be essentially symbolic if the issue was an FT boycott by the blind, given the smaller number of FT recipients to date.

Critically, it is the sighted who pioneered and now manage FT, and so assess the blind and their disability in a mainstream world of seeing people. We contend that identity and patient-centred meaning and valuing of the face are important sources to support arguments for FT for the blind. Ethicists can contribute by proposing a wider framework wherein disability and the blind person's experience of faces are well-founded considerations for medical practitioners and ethics boards in the process of FT decision-making.

Contributors $\mathrm{JL}$ is the sole author of the article.

Funding This research received no specific grant from any funding agency in the public, commercial or not-for-profit sectors.

Competing interests None declared.

Provenance and peer review Not commissioned; externally peer reviewed. 
(c) Article author(s) (or their employer(s) unless otherwise stated in the text of the article) 2018. All rights reserved. No commercial use is permitted unless otherwise expressly granted.

\section{REFERENCES}

1 Chim H, Amer H, Mardini S, et al. Vascularized composite allotransplant in the realm of regenerative plastic surgery. Mayo Clin Proc 2014;89:1009-20.

2 Siemionow MZ, Gordon CR. Institutional review board-based recommendations for medical institutions pursuing protocol approval for facial transplantation. Plast Reconstr Surg 2010;126:1232-9.

3 Bramstedt KA, Plock JA. Looking the world in the face: The benefits and challenges of facial transplantation for blind patients. Prog Transplant 2016;27:79-83.

4 Bueno EM, Diaz-Siso JR, Pomahac B. A multidisciplinary protocol for face transplantation at Brigham and Women's Hospital. J Plast Reconstr Aesthet Surg 2011;64:1572-9.

5 Carty MJ, Bueno EM, Lehmann LS, et al. A position paper in support of face transplantation in the blind. Plast Reconstr Surg 2012;130:319-24.

6 Pomahac B, Diaz-Siso JR, Bueno EM. Evolution of indications for facial transplantation. J Plast Reconstr Aesthet Surg 2011;64:1410-6.

7 Lemmens GM, Poppe C, Hendrickx H, et al. Facial transplantation in a blind patient: psychologic, marital, and family outcomes at 15 months follow-up. Psychosomatics 2015:56:362-70.

8 Welsh RL, Tuttle DW. Congenital and adventitious blindness. In: Moore JE, Graves WH, Patterson J, eds. Foundations of rehabilitation counseling with persons who are blind or visually impaired. New York: AFB Press, 1997:60-79.

9 Obasogie OK. Blinded by sight: seeing race through the eyes of the blind. Stanford: Stanford University Press, 2014:61.

10 Deshen S. Blind people: the private and public life of sightless Israelis. Albany, NY: SUNY Press, 1992:21.

11 Gregory R. The blind leading the sighted. Nature 2004;430:836.

12 Kleege G. Wearing the mask inside out. Soc Res 2000;67:47-59.

13 Kennedy JM. Drawings from Gaia, a blind girl. Perception 2003;32:321-40.
14 Herrmann U. A trojan horse of clay: art therapy in a residential school for the blind. Arts Psychother 1995;22:229-34.

15 Frith C, Law J. Cognitive and physiological processes underlying drawing skills. Leonardo 1995:28:203-5.

16 Magnusson A-K, Karlsson G. The body language of adults who are blind. Scand J Disabil Res 2008;10:71-89.

17 Cole J. About face. Cambridge, MA: MIT Press, 1998.

18 Kaplan-Myrth N. Alice without a looking glass: Blind people and body image. Anthropol Med 2000;7:277-99.

19 Ashikali EM, Dittmar H. Body image and restrained eating in blind and sighted women: A preliminary study. Body Image 2010;7:172-5.

20 Hammer G. Blind women's appearance management: negotiating normalcy between discipline and pleasure. Gender Soc 2012;26:406-32.

21 Fine I. Sensory systems: do you hear what I see? Nature 2014;508:461-2.

22 Linett M. Blindness and Intimacy in Early Twentieth-Century Literature. Mosaic 2013;46:27-42.

23 Kunz M, Faltermeier N, Lautenbacher S. Impact of visual learning on facial expressions of physical distress: a study on voluntary and evoked expressions of pain in congenitally blind and sighted individuals. Biol Psychol 2012;89:467-76.

24 Matsumoto D, Willingham B. Spontaneous facial expressions of emotion of congenitally and noncongenitally blind individuals. J Pers Soc Psychol 2009;96:1-10.

25 Rahman MM, Anam A, Yeasin M. E m o A s s i s t: emotion enabled assistive tool to enhance dyadic conversation for the visually impaired. Multimed Tools Appl 2017;:76:7699-730.

26 Widgerow AD, Chait LA. Aesthetic perspectives regarding physically and mentally challenged patients. Plast Reconstr Surg 2000;105:2251-4.

27 Burd A. Plastic surgery, body image and the blind. J Plast Reconstr Aesthet Surg 2007;60:1273-6.

28 Bramstedt KA. A lifesaving view of vascularized composite allotransplantation: patient experience of social death before and after face, hand, and larynx transplant. J Patient Exp 2017 [Epub ahead of print 6 Oct 2017].

29 Soares MO. Is the QALY blind, deaf and dumb to equity? NICE's considerations over equity. Br Med Bull 2012;101:17-31.

30 Blume S. The artificial ear: cochlear implants and the culture of deafness. New Brunswick, NJ: Rutgers University Press, 2010. 